\title{
Conhecimento, frequência e indicações de uso do Tratamento Restaurador Atraumático por cirurgiões-dentistas da rede municipal
de saúde de Passo Fundo, RS, Brasil
}

Knowledge, frequency, and indications for use of the Atraumatic Restorative Treatment by dental surgeons of the municipal health network of Passo Fundo, RS, Brazil

Sthefani Pinheiro*

Daniela Jorge Corralo*

Paulo do Prado Funk ${ }^{* *}$

\section{Resumo}

O Tratamento Restaurador Atraumático (TRA) possibilita o tratamento de cárie com o auxílio de instrumentos manuais (curetas odontológicas) e posteriormente a restauração da cavidade com cimento de ionômero de vidro (CIV). Na maioria dos casos, a anestesia e o isolamento absoluto são dispensados. Tal tratamento é realizado em locais onde o acesso a consultórios é impossibilitado ou quando o paciente não tem condições de receber o tratamento convencional. Objetivo: este estudo propôs verificar o conhecimento de Cirurgiões-Dentistas que trabalham na rede municipal de saúde de Passo Fundo, RS sobre o Tratamento Restaurador Atraumático (TRA), suas indicações e frequência de uso na rotina de trabalho clínico. Sujeitos e método: a amostra foi composta por 29 Cirurgiões-Dentistas que estavam trabalhando nas unidades de saúde durante $o$ período da coleta dos dados e responderam a um questionário sobre o TRA. Resultados: verificou-se que a maior parte dos profissionais que já ouviram falar sobre a técnica do TRA (93\%), conhecem suas indicações e tem conhecimento principalmente no que se refere aos materiais utilizados (85\%), no entanto, confundem o TRA com adequação do meio bucal. Um total de 21 CDs $(77,8 \%)$ relatam usar a técnica ou já terem utilizado no passado. Conclusão: conclui-se que os CDs conhecem as indicações do TRA, porém necessitam de maior conhecimento no que se refere aos aspectos que diferem o TRA da adequação do meio bucal e tratamento restaurador definitivo. A técnica é utilizada pelos profissionais principalmente no serviço público, com resultados satisfatórios, considerando-a eficaz para ser utilizada na rede de saúde.

Palavras-chave: Tratamento restaurador atraumático. Cimento ionômero de vidro. Cárie dentária.

\section{Introdução}

O interesse na prevenção e controle da cárie dentária aumentou nas últimas décadas. Isso se deve ao conhecimento da população por meio da divulgação da etiologia e dos fatores que contribuem para seu aparecimento e desenvolvimento. Apesar disso, principalmente em países subdesenvolvidos, dois terços da população mundial ainda apresentam sinais e sintomas dessa doença ${ }^{1}$. Embora o Tratamento Restaurador Atraumático (TRA) tenha sido desenvolvido para o emprego em comunidades desprovidas de recursos para a prática convencional, esse tem sido usado mesmo em locais nos quais a prática odontológica convencional é rotineira. Isso se deve ao baixo custo quando comparado a outros métodos, à praticidade da técnica e ao conforto proporcionado ao paciente, já que o uso da alta rotação e da anestesia é dispensada na maioria dos casos ${ }^{2}$. 
O tratamento restaurador atraumático consiste na remoção de tecido cariado, utilizando-se instrumentos cortantes manuais, e na restauração imediata da cavidade com cimento de ionômero de vidro (CIV), o qual é tido como material de escolha por possuir adesividade às estruturas dentais, dispensando a necessidade de desgaste dessas estruturas, bem como a característica de liberação de flúor e a sua biocompatibilidade ${ }^{3-4}$.

Na rede pública de saúde, a utilização de técnicas restauradoras atraumáticas que possam reduzir o estresse dos pacientes ao tratamento e, principalmente, o custo dos procedimentos deve ser preconizada $^{2,5}$.

Diante disso, verificou-se a necessidade de realizar um estudo para averiguar qual é o conhecimento dos profissionais sobre o TRA, suas indicações e frequência de uso na rotina de trabalho clínico, visto que ainda vivemos em uma realidade na qual muitas vezes não é possível realizar tratamentos convencionais.

\section{Sujeitos e método}

A pesquisa foi aprovada pelo Comitê de Ética em Pesquisa da Universidade de Passo Fundo com o número de protocolo C.A.A.E. 50025115.5.0000.5342.

\section{Local do estudo}

Este estudo ocorreu no município de Passo Fundo, RS, com cirurgiões- dentistas da rede municipal de saúde, que consentiram em participar do estudo por meio da assinatura do Termo de Consentimento Livre e Esclarecido (TCLE). Além disso, solicitou-se autorização da Secretaria de Saúde do município para a realização do estudo. O projeto foi enviado para apreciação do Comitê de Ética em Pesquisa (CEP) da Universidade de Passo Fundo (UPF) previamente à coleta dos dados.

\section{Coleta de dados}

Foi aplicado um questionário estruturado a Cirurgiões-Dentistas da rede municipal de saúde de Passo Fundo, com questões fechadas e abertas sobre o Tratamento Restaurador Atraumático (TRA). A aluna responsável pela pesquisa distribuiu os questionários nas unidades onde atuam os profissionais. O questionário propôs identificar qual é o conhecimento dos profissionais sobre o TRA, qual a frequência de uso da técnica e quais as indicações para a utilização dessa.

\section{Análise estatística}

Os dados coletados foram analisados por meio de estatística descritiva e pelo programa Excel.

\section{Resultados}

Foram distribuídos 44 questionários para os Cirurgiões-Dentistas que atuam nas Unidades Básicas de Saúde do município de Passo Fundo. Houve um retorno de 29 questionários.

A Tabela 1 mostra a distribuição dos cirurgiões-dentistas de acordo com a faixa etária.

Tabela 1 - Faixa etária dos CDs

\begin{tabular}{l|c}
\hline \multicolumn{1}{c|}{ Idade } & Frequência \\
\hline $21-26$ & 4 \\
$27-32$ & 11 \\
$33-39$ & 7 \\
Acima de 39 & 7 \\
Total CDs & 29 \\
\hline Fonte: elaboração dos autores.
\end{tabular}

Fonte: elaboração dos autores.

Foram entrevistados $11 \mathrm{CDs}$ do sexo masculino e 18 do sexo feminino, formados, em sua maior parte entre 2008 e 2013. A Figura 1 mostra a distribuição dos profissionais por ano de formatura.

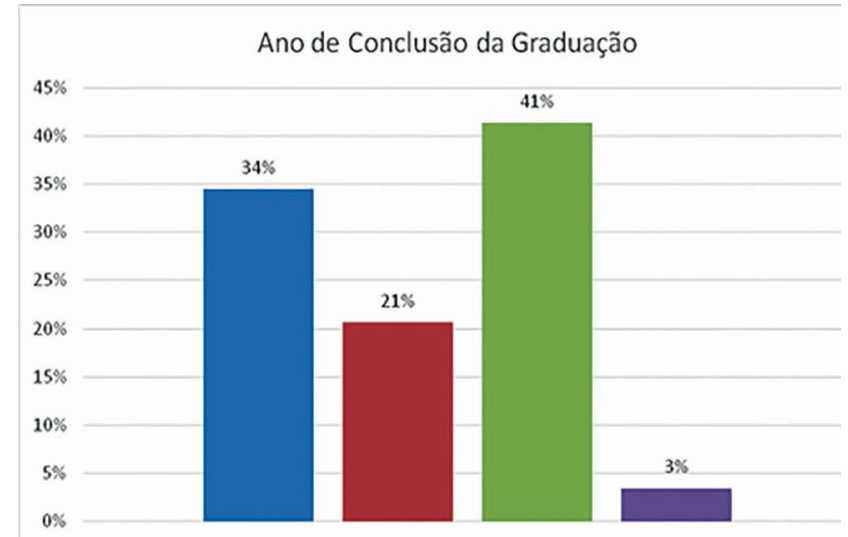

Figura 1 - Distribuição percentual dos CDs por ano de formatura Fonte: elaboração dos autores.

Dos questionários que retornaram, vinte e sete CDs (93\%) já haviam ouvido falar sobre o TRA, sendo que dois profissionais (7\%) nunca ouviram falar, não respondendo às demais questões do questionário. A principal fonte de obtenção de informações sobre a técnica foi durante a graduação.

A Tabela 2 mostra o conhecimento que os participantes possuem sobre o TRA. Para $67 \%$ dos cirurgiões-dentistas, o TRA é o mesmo que "adequação do meio bucal" e um terço dos profissionais consideram serem técnicas diferentes.

O TRA não é considerado um tratamento restaurador definitivo para 70\% dos CDs entrevistados. O ionômero de vidro é considerado por $85 \%$ dos profissionais como o material restaurador a ser utilizado. Para $41 \%$ dos CDs, a resina ou o IRM também poderiam ser usados no TRA, ao passo que 
59\% discordam da utilização desses dois materiais restauradores.

A utilização de equipamento elétrico para a realização de TRA não é considerada como necessária para $89 \%$ dos profissionais, ao passo que $11 \%$ consideram a necessidade de energia. A utilização de cureta para dentina, recortadores manuais, espátulas de cimento, placa de vidro e aplicador de material restaurador são considerados como necessários para a técnica atraumática para $81 \%$ dos CDs.

Tabela 2 - Conhecimento dos CDs sobre TRA $(n=27)$

\begin{tabular}{l|c|c}
\hline \multicolumn{1}{c|}{ Conhecimento } & Sim & Não \\
\hline TRA é o mesmo que adequação do meio bucal & $67 \%$ & $33 \%$ \\
TRA é um tratamento restaurador definitivo & $30 \%$ & $70 \%$ \\
Material restaurador utilizado TRA é ionômero & $85 \%$ & $15 \%$ \\
de vidro & $41 \%$ & $59 \%$ \\
Resina ou IRM também pode ser usado no TRA & $41 \%$ & $19 \%$ \\
Instrumentais para a realização do TRA & $89 \%$ & $11 \%$ \\
Não há necessidade de equipamento elétrico &
\end{tabular}

A Tabela 3 apresenta as indicações do TRA pelos Cirurgiões-Dentistas entrevistados.

Tabela 3 - Indicações do TRA pelos CDs $(n=27)$

\begin{tabular}{l|r|r}
\hline \multicolumn{1}{c|}{ Conhecimento } & \multicolumn{1}{c|}{ Sim } & \multicolumn{1}{c}{ Não } \\
\hline TRA pode ser usado para qualquer paciente & $93 \%$ & $7 \%$ \\
TRA é somente para dentição decídua & $15 \%$ & $85 \%$ \\
TRA é usado tanto em dentes anteriores & & \\
quanto posteriores & $93 \%$ & $7 \%$ \\
TRA não pode ser usado em exposição pulpar & $74 \%$ & $26 \%$ \\
\hline
\end{tabular}

Fonte: elaboração dos autores.

A utilização do TRA, para 93\% dos CDs, pode ser realizada em qualquer paciente, independente de idades ou condição de saúde, sendo que $85 \%$ dos profissionais reconhecem que a técnica pode ser utilizada em ambas as dentições, não apenas a decídua.

A utilização do Tratamento Restaurador Atraumático, tanto em dentes anteriores quanto em posteriores é possível para $93 \%$ dos CDs. A utilização do TRA em caso de exposição pulpar ou dor prolongada não é indicada para $74 \%$ dos profissionais.

A Tabela 4 mostra a frequência que os Cirurgiões-Dentistas possuem em relação à utilização da técnica do Tratamento Restaurador Atraumático.

Um total de $21 \mathrm{CDs}(77,8 \%)$ relataram usar a técnica ou já terem usado no passado, seja no serviço público ou consultório particular.
Tabela 4 - Frequência do uso do TRA pelos CDs $(n=27)$

\begin{tabular}{l|r|r}
\multicolumn{1}{c|}{ Experiência } & \multicolumn{1}{c|}{$\mathrm{n}$} & \multicolumn{1}{c}{$\%$} \\
\hline Usam atualmente & $\mathbf{1 9}$ & $\mathbf{7 0 , 4}$ \\
\hline Consultório particular & 0 & 0,0 \\
Serviço público & 13 & 68,4 \\
Ambos & 6 & 31,6 \\
\hline Usaram no passado & $\mathbf{2}$ & $\mathbf{7 , 4}$ \\
\hline Consultório particular & 0 & 0,0 \\
Serviço público & 2 & 100,0 \\
Ambos & 0 & 0,0 \\
\hline Nunca usaram & $\mathbf{6}$ & $\mathbf{2 2 , 2}$ \\
\hline Pretendem utilizar & 3 & 50,0 \\
Não pretendem utilizar & 3 & 50,0 \\
\hline Fonte: elaboração dos autores. & &
\end{tabular}

Entre os que já utilizaram o TRA, 52,4\% obtiveram resultados satisfatórios, $33,3 \%$ obtiveram resultados relativamente satisfatório se $14,3 \%$ não souberam responder que resultados obtiveram. Nenhum dos CDS considerou os resultados insatisfatórios (Tabela 5).

Tabela 5 - Resultados dos CDs que utilizaram TRA ( $n=21)$

\begin{tabular}{l|r|r}
\hline \multicolumn{1}{c|}{ Resultado obtido com o uso do TRA } & \multicolumn{1}{c|}{ n } & \multicolumn{1}{c}{$\%$} \\
\hline Satisfatório & 11 & 52,4 \\
Relativamente satisfatório & 7 & 33,3 \\
Insatisfatório & 0 & 0 \\
Não sabe & 3 & 14,3 \\
\hline Fonte: elaboração dos autores.
\end{tabular}

Com relação à eficácia do Tratamento Restaurador Atraumático (Tabela 6), a técnica é considerada eficaz para 81,5\% dos 27 CDs entrevistados.

As justificativas apresentadas para a percepção da eficácia foram: facilidade da técnica por não utilizar eletricidade e reduzir a ansiedade do paciente $(33,4 \%)$, permitir fazer a adequação do meio bucal $(31,8 \%)$, preservar a estrutura dental $(13,6 \%)$. Para a eficácia do TRA $18,2 \%$ dos CDs a consideram desde que os pacientes recebam orientações.

Tabela 6 - Eficácia do TRA entre os CDs

\begin{tabular}{|c|c|c|}
\hline Acredita na eficácia do TRA & $\mathrm{n}$ & $\%$ \\
\hline Sim & 22 & 81,5 \\
\hline Justificativa & & \\
\hline $\begin{array}{l}\text { Facilidade da técnica, não utiliza } \\
\text { eletricidade e reduz a ansiedade do } \\
\text { paciente }\end{array}$ & 8 & 33,4 \\
\hline Permite fazer a adequação do meio bucal. & 7 & 31,8 \\
\hline $\begin{array}{l}\text { Desde que os pacientes recebam } \\
\text { orientação. }\end{array}$ & 4 & 18,2 \\
\hline Preservar a estrutura dental. & 3 & 13,6 \\
\hline
\end{tabular}

Fonte: elaboração dos autores. 
A Tabela 7 mostra que a eficácia do TRA é duvidosa para $18,5 \%$ dos profissionais, justificando a possibilidade de recidiva de cárie dentária $(80,0 \%$ dos $\mathrm{CDs}$ ) e dúvida quanto à remoção completa da dentina cariada $(20,0 \%)$. Nenhum dos respondentes acha a técnica ineficaz.

Tabela 7 - Eficácia do TRA entre os CDs

\begin{tabular}{l|r|c}
\hline \multicolumn{1}{c|}{ Acredita na eficácia do TRA } & \multicolumn{1}{c|}{$\mathrm{n}$} & \multicolumn{1}{c}{$\%$} \\
\hline Tem dúvida & 5 & 18,5 \\
Justificativa & & \\
$\begin{array}{l}\text { A dentina cariada deveria ser removida } \\
\text { completamente. }\end{array}$ & 1 & 20,0 \\
$\begin{array}{l}\text { Dúvida se irá ocasionar recidiva. } \\
\text { Fonte: elaboração dos autores. }\end{array}$ & 4 & 80,0 \\
\hline
\end{tabular}

A maioria dos profissionais entrevistados (81,5\%) acreditam que o TRA deve ser utilizado no serviço público, justificando: ter apresentado bons resultados clínicos $(31,8 \%)$, devido ao baixo custo, não necessitar de instrumentais diferenciados e diminuir o tempo clínico $(27,3 \%)$, reduzir o estresse do paciente e encaminhamento para endodontia e exodontia $(22,7 \%)$. Por fim, $13,6 \%$ dos cirurgiões-dentistas acreditam que deve ser utilizado principalmente em dentes decíduos e 4,5\% acham que deve ser utilizado pelo fato de preservar o elemento dental. Os dados podem ser vistos na Tabela 8.

Tabela 8 - Realização do TRA no Serviço Público

\begin{tabular}{l|r|r}
\hline \multicolumn{1}{c|}{ Utilização do TRA no Serviço Público } & \multicolumn{1}{c|}{ n } & \multicolumn{1}{c}{$\%$} \\
\hline SIM & 22 & 81,5 \\
Justificativa & 7 & 31,8 \\
$\begin{array}{l}\text { Apresenta bons resultados clínicos. } \\
\begin{array}{l}\text { Baixo custo, não necessita de instrumentais } \\
\text { diferenciados e diminui o tempo clínico. }\end{array}\end{array}$ & 6 & 27,3 \\
$\begin{array}{l}\text { Reduz o estresse dos pacientes e } \\
\text { encaminhamento para endodontia e } \\
\text { exodontia. }\end{array}$ & 5 & 22,7 \\
$\begin{array}{l}\text { Principalmente em dentes decíduos } \\
\text { Preserva o elemento dental. }\end{array}$ & 3 & 13,6 \\
\hline
\end{tabular}

Fonte: elaboração dos autores.

As dúvidas quanto à utilização do TRA no serviço público é citada por $14,8 \%$ dos Profissionais $(\mathrm{n}=4)$, pelo fato de não saber se o paciente irá retornar para finalizar o tratamento. Apenas um respondente $(3,7 \%)$ acha o método impróprio para a utilização no serviço público pelo fato de não ter material e instrumental adequado.

Por fim, quando questionados se havia interesse dos participantes em saber mais informações ou aprimoramento sobre o TRA, $81 \%$ dos CDs afirmam interesse e $19 \%$ relatam não ter interesse.

\section{Discussão}

O tratamento restaurador atraumático foi desenvolvido com o objetivo de ser utilizado em comunidades carentes. Apesar disso, o uso de TRA tem ocorrido em países mais desenvolvidos, o que se justifica pelo fato de esse auxiliar no tratamento odontológico precoce em bebês; proporcionar maior conforto para pacientes extremamente ansiosos ou pacientes especiais por dispensar o uso da alta rotação e da anestesia; ser utilizado em crianças com alto risco de cárie; oferecer praticidade técnica e baixo custo quando comparado a outros métodos restauradores mais convencionais ${ }^{2}$.

Quanto ao conhecimento dos profissionais da rede municipal de saúde de Passo Fundo-RS sobre o TRA, verificou-se que a maioria já ouviu falar sobre a técnica, principalmente na graduação. Assim como no estudo feito com docentes de 39 faculdades do Brasil, no qual os dados coletados mostram que $100 \%$ desses cursos abordam conteúdos teórico-práticos sobre TRA, principalmente nas disciplinas de Saúde Coletiva e Odontopediatria ${ }^{6}$.

Dos profissionais da rede pública entrevistados, $70 \%$ não consideram o TRA como definitivo, pois para $67 \%$ dos CDs ele é o mesmo que adequação do meio bucal. Isso pode ser explicado pelo fato de os procedimentos possuírem grande semelhança. Ambas as técnicas não necessitam de anestesia local e alta rotação, o material restaurador é o mesmo e, na maioria das vezes, os mesmos instrumentais são utilizados, mas as finalidades de cada técnica são distintas. O TRA é considerado como um tratamento restaurador definitivo ${ }^{5,7,8}$

Em relação ao material restaurador utilizado no TRA, 85\% dos profissionais pesquisados usam o ionômero de vidro e $41 \%$ dos CDs afirmam que resina composta ou IRM pode ser o material utilizado no tratamento restaurador atraumático. No entanto, sabe-se que a técnica preconiza o uso de cimento ionômero de vidro, por proporcionar um bom selamento da cavidade e, também, pela sua característica de liberar flúor'.

Ainda se tratando do conhecimento dos CDs entrevistados, $81 \%$ deles concordam que para a realização do TRA são necessários os seguintes instrumentais: cureta para dentina, recortadores, espátula, placa de vidro e aplicador, ao passo que 19\% acreditam que a técnica não é realizada com esses instrumentais. A necessidade de equipamentos elétricos não é necessária para $89 \%$ dos profissionais e, para $11 \%$, a energia elétrica não pode ser dispensada. Alguns autores confirmam que esses instrumentais são os mais adequados e equipamentos elétricos não são necessários para a realização da técnica ${ }^{10}$.

No que se refere às indicações do TRA, 93\% dos CDs concordam que pode ser indicado para qualquer paciente (bebês, crianças, adolescentes, adultos, portadores de necessidades especiais, gestantes 
e idosos) e apenas $7 \%$ acreditam que sua indicação não é possível para qualquer paciente. Para $85 \%$ dos CDs, é reconhecido o uso do TRA em ambas as dentições, não apenas na dentição decídua. A utilização do TRA, tanto em dentes anteriores quanto em posteriores é possível para 93\% dos entrevistados. O uso da técnica em caso de dor prolongada ou exposição pulpar não é indicada para $74 \%$ dos entrevistados. Para alguns autores, esta técnica pode ser empregada em quaisquer pacientes que apresentam dentes decíduos e permanentes com lesões de cárie em dentina tanto em dentes anteriores quanto posteriores e que seu uso deve ser em dentes sem alteração pulpar irreversível, ou seja, sem sinais ou sintomas como história de dor ${ }^{5-6}$. Chama a atenção que, para $26 \%$ dos entrevistados, isso não impede a indicação de tal técnica.

Com relação à frequência dos profissionais de Passo Fundo-RS que já utilizaram a técnica $(77,8 \%)$, a maioria considera satisfatória $(52,4 \%)$ e, relativamente, satisfatória $(33,3 \%)$. É interessante notar que os CDs de Passo Fundo-RS, utilizaram não apenas no serviço público, mas, também, no consultório particular, o que confirma que a sua aplicação não é adequada apenas para populações de baixa renda e com poucos recursos tecnológicos, mas em todos os segmentos socioeconômicos ${ }^{2}$. Há uma pequena parcela que nunca utilizou o TRA $(22,2 \%)$, sendo que alguns têm interesse em aderir à técnica e outros não tem interesse em utilizá-la. Talvez isso possa ser justificado pelo fato de não haver incentivo para o conhecimento e maior preparo técnico para a realização de procedimentos diferenciados ${ }^{8}$.

Dos CDs entrevistados $81,5 \%$ acreditam na sua eficácia, percentual melhor do que os $60 \%$ encontrados num trabalho que pesquisou CDs de Goiânia ${ }^{8}$. As justificavas apresentadas pelos profissionais de Passo Fundo referem-se à facilidade da técnica por não utilizar eletricidade e reduzir a ansiedade do paciente $(33,4 \%)$, permitir fazer a adequação do meio bucal $(31,8 \%)$, preservar a estrutura dental $(13,6 \%)$ ou desde que os pacientes recebam orientações $(18,2 \%)$. Com tais justificativas, percebe-se que os profissionais tem um conhecimento considerável sobre TRA, porém, novamente, o confundiram com adequação do meio bucal. O TRA é considerado como um tratamento restaurador definitivo, diferente de adequação do meio bucal ${ }^{7}$. O TRA é considerado duvidoso para 18,5\% dos profissionais, justificando a possibilidade de recidiva de cárie dentária (80,0\% dos CDs) e dúvida quanto à remoção completa da dentina cariada $(20,0 \%)$. No entanto, com o princípio da abordagem de mínima intervenção, há evidência de que a remoção completa da dentina cariada em lesões profundas de dentina não é requerida para o sucesso do tratamento ${ }^{6}$. Nenhum entrevistado considera a técnica ineficaz.

A utilização do TRA no serviço público é considerada importante para $81,5 \%$ dos CDs, justificando para isso os bons resultados clínicos apresentados, o baixo custo, a não necessidade de instrumentais diferenciados, a redução do tempo clínico e estresse dos pacientes e a preservação do elemento dental. Assim como alguns autores que consideram seu uso devido a essas vantagens ${ }^{2,8,10}$.

\section{Conclusão}

Verificou-se que a maioria dos profissionais conheceu a técnica do tratamento restaurador atraumático durante a graduação e conhecem as suas indicações. A pesquisa demonstrou que os CDs necessitam de maior conhecimento no que se refere aos aspectos que diferem o TRA da adequação do meio bucal e tratamento restaurador definitivo. A técnica é utilizada pelos profissionais principalmente no serviço público, com resultados satisfatórios, considerando-a eficaz para ser utilizada na rede de saúde. Finalmente, sugere-se uma educação continuada sobre tratamento restaurador atraumático para os cirurgiões-dentistas da rede pública do município.

\section{Abstract}

The Atraumatic Restorative Treatment (ART) allows treating caries with the aid of hand tools (dental curette), and then restoring the cavity with glass ionomer cement (GIC). In most cases, anesthesia and absolute isolation are dismissed. This treatment is performed in places with no access to dental offices or when the patient is unable to receive conventional treatment. Objective: this study aimed to verify the knowledge of dental surgeons working in the municipal health network of Passo Fundo, RS, Brazil, on the Atraumatic Restorative Treatment $(A R T)$, as well as its indications and frequency of use in the clinical routine. Subjects and method: the sample was composed of 29 dentists working in health facilities during the period of data collection, who answered a questionnaire on ART. Results: it was found that most professionals who have heard of the ART technique $(93 \%)$ know their indications, especially regarding the materials used (85\%). However, these professionals mistake ART for the adequacy of the oral environment. A total of 21 dentals surgeons $(77.8 \%)$ report using the technique or having used it in the past. Conclusion: it is concluded that dental surgeons know the indications of $A R T$, but need more knowledge regarding the aspects that differ ART from the adequacy of the oral environment and definitive restorative treatment. The technique is mainly used by professionals in the public service, showing satisfactory results that make it effective for use in the health network.

Keywords: Atraumatic restorative treatment. Glass ionomer cement. Dental cavity. 


\section{Referências}

1. Frencken JE, Holmgren CJ. How effective is ART in the management of dentalcaries? Community Dent Oral Epidemiol, 1999; 27(6):423-30.

2. Mickenautsch S, Rudolph M, Ogunboede E, Frencken J. The impact of the ART approach on the treatment profile in a Mobile Dental System in South Africa. Int Dent. 1999; 49(3):132-8

3. Martins AR, Ferreira AS. Tratamento restaurador atraumático: uma revisão de literatura.Trabalho de graduação (Odontologia) Faculdade de Ciências da Saúde, Universidade do Vale do Paraíba, São José dos Campos, 2001.

4. Figueiredo KSP, Forte FDS, Sampaio FC. Desempenho clínico de restaurações ART (tratamento restaurador atraumático) em crianças atendidas na clínica de cariologia da UFPB. Rev Odontol Unesp, 2008;37(4);351-5.

5. Monnerat FA. Tratamento Restaurador Atraumático - Abordagem Clínica em Saúde Pública. Ed. Elsevier Medicina Brasil, 2015; 02-10.

6. Massara ML, Wambier DS, Imparato JC. Tratamento restaurador atraumático. Manual de Referência para Procedimentos Clínicos em Odontopediatria, 2010. 156-63.

7. Oliveira L, Neves A, Neves M, Souza I. Tratamento Restaurador Atraumático e Adequação do Meio Bucal; 1998. p. 94-9.

8. Rios LE, Essado REP, Freire MCM. Tratamento restaurador atraumático: conhecimentos e atitudes de CD's do servico público de Goiânia-GO. Rev Odontol UNESP 2006;50(3):75-80.

9. Luz PB. Avaliação de duas técnicas restauradoras de mínima intervenção em lesões cariosas profundas de molares decíduos. (Dissertação de Mestrado). Faculdade de Odontologia, Universidade Federal do Rio Grande do Sul, Porto Alegre; 2009.

10. Monico M, Tostes M. Tratamento restaurador simplificado para atendimento infantil. JBP 1998;1(4):9-11.

\section{Endereço para correspondência:}

Sthefani Pinheiro

Rua Ângelo Tonial, 230, Centro

99925000 Ipiranga do Sul, RS

Fone (54) 9137-1779

E-mail: sthefani_pinheiro@hotmail.com

Recebido: 06/07/2016. Aceito: 20/03/2017. 